\title{
Proportional Fair Coding for 802.11 WLANs
}

\author{
Xiaomin Chen, Douglas Leith, \\ Hamilton Institute, NUI Maynooth
}

\begin{abstract}
We consider an 802.11 WLAN with lossy links and flow delay deadlines and we derive the joint allocation of coding rate and airtime that achieves the proportionally fair throughput allocation. The noisy wireless links are modelled as symbollevel binary symmetric channels (BSCs). We show that the joint optimisation decomposes into decoupled allocation tasks, i.e. partitioning into layers is optimal, and the optimal allocation assigns equal total air-times to flows.
\end{abstract}

\section{INTRODUCTION}

Proportional fairness in 802.11 WLANs has been the subject of a considerable body of literature. The CSMA/CA scheduling used in 802.11 differs fundamentally from wired and TDMA wireless networks due to the carrier sense deferral of contention window countdown and the occurrence of collisions, both of which lead to the coupling of station transmissions within a WLAN and the rate region being nonconvex. Hence, well established utility fairness techniques from wired networks cannot be directly applied to random access CSMA/CA wireless networks.

Recently, [8] provides the first rigorous analysis of proportional fairness in 802.11 WLANs and in this paper we extend that work in a number of different directions. Firstly, the analysis in [8] assumes that transmissions are loss-free while in this paper we relax this assumption. Secondly, building upon the work in [2], we view the channel provided by the WLAN as a binary symmetric channel (BSC), as opposed to a more conventional packet erasure channel (PEC). That is, rather than simply discarding corrupted frames, we consider viewing a received frame as a binary vector in which an unknown subset of bits have been "flipped". Thirdly, we extend the analysis in [8] to allow delay deadline constraints to be included. Such delay constraints are especially relevant for delay-sensitive traffic such as video and voice but are also important for data traffic as delay affects flow completion times. Our analysis builds on the approach in [6] for TDMA wireless networks.

We show that the joint optimisation of coding rate and airtime decomposes into decoupled allocation tasks, i.e. partitioning into layers is optimal. This property of 802.11 differs from and contrasts with the results in [6] for TDMA networks. Further, we establish that the proportional fair coding rate and airtime allocation (i) assigns equal total airtime (i.e. airtime including both successful and failed transmissions) to every station in a WLAN, (ii) the station airtimes sum to unity (ensuring operation at the rate region boundary), and (iii) the optimal coding rate is selected to maximise goodput (treating packets decoded after the delay deadline as losses).

Supported by Science Foundation Ireland grant 07/IN.1/I901.

\section{NETWORK MODEL}

We consider an 802.11 WLAN with $n \geq 2$ stations. We consider per station throughput fairness and so define a flow to be a sequence of packets transmitted from the same station. Let $\mathcal{F}$ denote the set of flows, with $|\mathcal{F}|=n$.

1) BSC channel: The channel provided by the WLAN is modelled as a binary symmetric channel (BSC). That is, rather than simply discarding corrupted frames at the receiver, we view a decoded frame above PHY layer as a binary vector in which an unknown subset of bits have been "flipped". Let $\alpha_{f}$ denote the crossover probability for flow $f$, i.e. a bit of flow $f$ is corrupted with probability $\alpha_{f}$. For $802.11 \mathrm{a} / \mathrm{g}$, experimental measurements [1] and theoretical analysis [2] indicate that even with a $10-30 \%$ packet erasure rate typically only a small fraction (usually $<1 \%$ ) of bits within corrupted packets are in error. Thus, although noisy, the corrupted packets potentially provide a reasonable channel through which we can transmit information.

2) Decoding error probability: To protect against errors when recovering information symbols, a linear maximumdistance separable (MDS) code [4] is used at the MAC layer for additional forward error correction. Information frames of flow $f \in \mathcal{F}$ are assumed of size $k_{f}$ symbols. Information symbols are formed into blocks of $D_{f} k_{f}$ symbols, where $D_{f} \in \mathbb{Z}^{+}$is the number of frames that the block may span. Each block of $D_{f} k_{f}$ information symbols is encoded into a block of $D_{f} l_{f}$ coded symbols, with the coding rate $r_{f}=k_{f} / l_{f}, 0<r_{f} \leq 1 . l_{f}$ is the number of coded symbols in a transmitted packet, i.e. the transmitted packet size. We assume that the network limits the packet size to be no more than a maximal value $\bar{l}_{f}$ e.g, the 802.11 standard limits the maximum packet size to be $2304 \mathrm{~B}$. The quantity $D_{f}$ is a quality of service parameter. It specifies the decoding delay deadline for flow $f$, i.e. the receiving station must decode an information packet of flow $f$ after collecting at most $D_{f}$ successive coded packets.

It is shown in [6] that in a BSC the probability of a decoding error for flow $f$ is upper bounded by

$$
e_{f}=\exp \left(-D_{f} l_{f}\left[\theta_{f} v_{f}-\log \left(1-\beta_{f}+\beta_{f} e^{\theta_{f}}\right)\right]\right)
$$

where $\beta_{f}=1-\left(1-\alpha_{f}\right)^{m}$ is the symbol error probability with $m \geq 1$ being the number bits per MDS code symbol, $v_{f}:=\frac{1-r_{f}}{2}, 0 \leq v_{f}<\frac{1}{2}$ and $\theta_{f}>0$ is the Chernoff-bound parameter. This bound is valid for all $\theta_{f}>0$, and the tightest bound is obtained by minimising $e_{f}$ for $\theta_{f}>0$. This optimised bound is asymptotically tight [6], and thus the network utility maximisation problem can be posed based on this bound.

3) Station throughput: We assume that the RTS/CTS mechanism is used to make fast recovery from collisions. On a 
collision only a pair of RTS/CTS packets are sent. Each 802.11 MAC time slot may be a PHY idle slot, a successful transmission or a colliding transmission. Let $\tau_{f}$ denote the probability that the station carrying flow $f$ attempts to transmit in a given MAC slot. The probability that a time slot is idle is $P_{i}=\prod_{f \in \mathcal{F}}\left(1-\tau_{f}\right)$. The probability that the station carrying flow $f$ makes a successful transmission is $P_{s, f}=\tau_{f} \prod_{g \in \mathcal{F}, g \neq f}\left(1-\tau_{g}\right)=\frac{\tau_{f}}{1-\tau_{f}} P_{i}$. The probability that a time slot is a successful transmission is $P_{s}=\sum_{f \in \mathcal{F}} P_{s, f}=$ $P_{i} \sum_{f \in \mathcal{F}} \frac{\tau_{f}}{1-\tau_{f}}$. The throughput of flow $f$ is given by

$$
s_{f}=\frac{P_{s, f} l_{f}}{\sigma P_{i}+\sum_{f \in \mathcal{F}} T_{s, f} P_{s, f}+T_{c}\left(1-P_{i}-P_{s}\right)}
$$

in which $\sigma$ is the duration of a PHY idle slot, $T_{c}=T_{R T S}+$ $T_{S I F S}+T_{C T S}+T_{D I F S}$ is the duration of a collision and $T_{s, f}$ is the duration of a successful transmission by flow $f$. Note that $T_{c}$ is the same for all flows due to the use of RTS/CTS handshaking but we do not assume that flows use the same packet successful transmission duration $T_{s, f}$.

We relate packet successful transmission duration $T_{s, f}$ to the packet size $l_{f}$ via the PHY rate $w_{f}$ of flow $f$. Namely, $T_{s, f}=\frac{l_{f}}{w_{f}}+T_{o}$ where $T_{o}$ is the 802.11 protocol overhead associated with each successful transmission and is given by

$T_{o}=T_{R T S}+T_{C T S}+3 \times T_{S I F S}+T_{P H Y h d r}+T_{A C K}+T_{D I F S}$

Letting $x_{f}=\tau_{f} /\left(1-\tau_{f}\right)$ the throughput expression can be rewritten as

$$
s_{f}=\frac{x_{f} l_{f}}{X T_{c}}
$$

where $X=\frac{\sigma}{T_{c}}+\sum_{f \in \mathcal{F}}\left(\frac{l_{f}}{w_{f} T_{c}}+\frac{T_{o}}{T_{c}}-1\right) x_{f}+\prod_{f \in \mathcal{F}}(1+$ $\left.x_{f}\right)-1$. When all flows use the same packet size and PHY rate, throughput expression (1) is identical to that previously used in [3], [8].

4) Notation: We use bold notation to indicate a vector, thus $\boldsymbol{l}:=\left[l_{f}\right]_{f \in \mathcal{F}}$ is the vector of packet sizes, $\boldsymbol{x}:=\left[x_{f}\right]_{f \in \mathcal{F}}$ the vector of station attempt rates, $\boldsymbol{\theta}:=\left[\theta_{f}\right]_{f \in \mathcal{F}}$ the vector of Chernoff parameters, $\boldsymbol{v}:=\left[v_{f}\right]_{f \in \mathcal{F}}$ the vector of coding rates.

\section{PROPORTIONAL FAIR ALlOCATION}

We find the proportional fair rate allocation by solving

$$
\max _{\boldsymbol{l}, \boldsymbol{v}, \boldsymbol{x}, \boldsymbol{\theta}} U(\boldsymbol{l}, \boldsymbol{v}, \boldsymbol{x}, \boldsymbol{\theta}):=\sum_{f \in \mathcal{F}} \log g_{f}
$$

s.t. $0<l_{f} \leq \bar{l}_{f}, \theta_{f}>0, x_{f}>0,0 \leq v_{f}<1 / 2 \forall f \in \mathcal{F}$

where $g_{f}=s_{f}\left(1-2 v_{f}\right)\left(1-e_{f}\left(l_{f}, v_{f}, \theta_{f}\right)\right)$ is the goodput of flow $f \in \mathcal{F}$ after decoding. While the constraints are convex, it can be verified that $\log \left(1-e_{f}\left(l_{f}, v_{f}, \theta_{f}\right)\right)$ is not jointly concave in $\left(\theta_{f}, l_{f}, v_{f}\right)$ and so the maximisation problem is not a standard convex optimisation task. We proceed by carrying out the optimisation sequentially i.e. first finding $\boldsymbol{\theta}^{*}=\underset{\boldsymbol{\theta}}{\arg \max } U(\boldsymbol{l}, \boldsymbol{v}, \boldsymbol{x}, \boldsymbol{\theta})$ and then $\max _{\boldsymbol{l}, \boldsymbol{v}, \boldsymbol{x}} U\left(\boldsymbol{l}, \boldsymbol{v}, \boldsymbol{x}, \boldsymbol{\theta}^{*}\right)$. By [6, Lemma 3], provided $U(\boldsymbol{l}, \boldsymbol{v}, \boldsymbol{x}, \boldsymbol{\theta})$ is concave in $\boldsymbol{\theta}$ and $U\left(\boldsymbol{l}, \boldsymbol{v}, \boldsymbol{x}, \boldsymbol{\theta}^{*}\right)$ is jointly concave in $(\log \boldsymbol{l}, \log \boldsymbol{v}, \log \boldsymbol{x})$, the solution to the sequential optimisation is the unique solution to the original optimisation.

\section{A. Optimal Chernoff parameter $\theta_{f}^{*}$}

We begin by considering the optimisation

$$
\max _{\boldsymbol{\theta}} U(\boldsymbol{l}, \boldsymbol{v}, \boldsymbol{x}, \boldsymbol{\theta}) \quad \text { s. t. } \quad \theta_{f}>0 \quad \forall f \in \mathcal{F}
$$

It can be verified that $U$ is concave in $\boldsymbol{\theta}$ ( $U$ is separable in the $\theta_{f}$ 's and it can be verified by inspection of the second derivative that $U$ is concave for each $\theta_{f}$ ). Hence, the problem is a convex and from the KKT conditions the optimal $\theta_{f}$ is

$$
\theta_{f}^{*}\left(v_{f}\right)=\log \left(\frac{v_{f}}{\beta_{f}}\right)-\log \left(\frac{1-v_{f}}{1-\beta_{f}}\right)
$$

Observe that when $v_{f} \leq \beta_{f}, \theta_{f} \leq 0$. To ensure $\theta_{f}>0$, it is required that $v_{f}>\beta_{f}$.

\section{B. Optimal airtime, coding rate and transmit rate $l_{f}^{*}, v_{f}^{*}, x_{f}^{*}$}

We now solve

$$
\begin{array}{ll} 
& \max _{\boldsymbol{l , v , \boldsymbol { x }}} U\left(\boldsymbol{l}, \boldsymbol{v}, \boldsymbol{x}, \boldsymbol{\theta}^{*}(\boldsymbol{v})\right) \\
\text { s.t } & 0<l_{f} \leq \bar{l}_{f}, x_{f}>0, \beta_{f}<v_{f}<1 / 2 \quad \forall f \in \mathcal{F}
\end{array}
$$

Unfortunately $U\left(\boldsymbol{l}, \boldsymbol{v}, \boldsymbol{x}, \boldsymbol{\theta}^{*}\right)$ is not jointly concave in $\boldsymbol{l}, \boldsymbol{v}, \boldsymbol{x}$. We therefore change to work in terms of log-transformed variables $\tilde{l}_{f}=\log \left(l_{f}\right), \tilde{I}_{f}=\log \left(I_{f}\right)$ and $\tilde{x}_{f}=\log \left(x_{f}\right)$ where

$$
I_{f}\left(v_{f}\right):=v_{f} \log \left(\frac{v_{f}}{\beta_{f}}\right)+\left(1-v_{f}\right) \log \left(\frac{1-v_{f}}{1-\beta_{f}}\right)
$$

Since $\tilde{I}_{f}\left(v_{f}\right)$ is a monotone increasing function of $v_{f}$ (verified by inspection of first derivative), the inverse mapping from $\tilde{I}_{f}$ to $v_{f}$ exists and is one-to-one i.e. we can work interchangeably with either $v_{f}$ or $\tilde{I}_{f}$. With the obvious abuse of notation we use $v_{f}\left(\tilde{I}_{f}\right)$ to denote the $v_{f}$ value corresponding to a value of $\tilde{I}_{f}$. Note that $I_{f}$ arises naturally in our problem since $e_{f}\left(l_{f}, v_{f}, \theta_{f}^{*}\left(v_{f}\right)\right)=e^{-D_{f} l_{f} I_{f}\left(v_{f}\right)}$. The optimisation becomes

$$
\begin{array}{ll} 
& \max _{\tilde{\boldsymbol{l}}, \tilde{\boldsymbol{I}}, \tilde{\boldsymbol{x}}} U_{1}(\tilde{\boldsymbol{l}}, \tilde{\boldsymbol{I}}, \tilde{\boldsymbol{x}}):=U\left(e^{\tilde{\boldsymbol{l}}}, \boldsymbol{v}(\tilde{\boldsymbol{I}}), e^{\tilde{\boldsymbol{x}}}, \boldsymbol{\theta}^{*}(\boldsymbol{v}(\tilde{\boldsymbol{I}}))\right) \\
\text { s.t } & \tilde{l}_{f} \leq \log \bar{l}_{f}, \beta_{f}<v_{f}\left(\tilde{I}_{f}\right)<1 / 2 \quad \forall f \in \mathcal{F}
\end{array}
$$

Importantly, we have the following lemma:

Lemma 1: $U_{1}(\tilde{\boldsymbol{l}}, \tilde{\boldsymbol{I}}, \tilde{\boldsymbol{x}})$ is jointly concave in $\tilde{\boldsymbol{l}}, \tilde{\boldsymbol{I}}, \tilde{\boldsymbol{x}}$.

Proof:

$$
\begin{aligned}
U_{1}(\tilde{\boldsymbol{l}}, \tilde{\boldsymbol{I}}, \tilde{\boldsymbol{x}})= & \sum_{f \in \mathcal{F}} \log s_{f}+\log \left(1-2 v_{f}\right)+\log \left(1-e_{f}\right) \\
= & \sum_{f \in \mathcal{F}} \tilde{x}_{f}-\log X+\tilde{l}_{f}-\log T_{c}+\log \left(1-2 v_{f}\left(\tilde{I}_{f}\right)\right) \\
& +\log \left(1-e^{-D_{f} e^{\tilde{l}_{f}+\tilde{I}_{f}}}\right)
\end{aligned}
$$

By [6, Lemma 4], the last two terms in $U_{1}$ are jointly concave in $\left(\tilde{l}_{f}, \tilde{I}_{f}\right)$. It remains to show that $\log X$ is jointly convex in $\tilde{\boldsymbol{l}}, \tilde{\boldsymbol{x}}$. Now,

$$
\begin{aligned}
& X=\frac{\sigma}{T_{c}}+\sum_{f \in \mathcal{F}}\left(\frac{e^{\tilde{l}}}{T_{c} w_{f}}+\frac{T_{o}}{T_{c}}-1\right) e^{\tilde{x}_{f}}+\prod_{f \in \mathcal{F}}\left(1+e^{\tilde{x}_{f}}\right)-1 \\
& =\frac{\sigma}{T_{c}}+\frac{1}{T_{c} w_{f}} \sum_{f \in \mathcal{F}} e^{\tilde{l}} e^{\tilde{x}_{f}}+\frac{T_{o}}{T_{c}} \sum_{f \in \mathcal{F}} e^{\tilde{x}_{f}}+\sum_{k=2}^{N} \sum_{A \subseteq \mathcal{F},|A|=k} \prod_{j \in A} e^{\tilde{x}_{j}}
\end{aligned}
$$


Since the logarithm of a sum of exponentials is a convex function [7], it follows that $\log X$ is convex in the transformed variables $\tilde{\boldsymbol{l}}, \tilde{\boldsymbol{x}}$.

By Lemma 1, the transformed maximisation problem is a convex optimisation problem. The Lagrangian is

$$
L=U_{1}-\sum_{f \in \mathcal{F}} p_{f}\left(\tilde{l}_{f}-\log \bar{l}_{f}\right)
$$

where multiplier $p_{f} \geq 0$ and we have dropped terms involving the constraints on $v_{f}$ in order to streamline notation and because these are almost never active in practical cases. The KKT optimality conditions corresponding to $\tilde{x}_{f}, \tilde{I}_{f}$ and $\tilde{l}_{f}$ are

$$
\begin{aligned}
& \frac{n x_{f}}{X}\left(\frac{l_{f}}{T_{c} w_{f}}+\frac{T_{o}}{T_{c}}-1+\prod_{g \in \mathcal{F}, g \neq f}\left(1+x_{g}\right)\right)=1 \\
& \frac{2}{1-2 v_{f}}=\frac{e_{f}\left(l_{f}, v_{f}\right)}{1-e_{f}\left(l_{f}, v_{f}\right)} D_{f} l_{f} \theta_{f}^{*}\left(v_{f}\right) \\
& 1-\frac{n x_{f}}{X} \frac{l_{f}}{T_{c} w_{f}}+\frac{e_{f}\left(l_{f}, v_{f}\right)}{1-e_{f}\left(l_{f}, v_{f}\right)} D_{f} l_{f} I_{f}\left(v_{f}\right)-p_{f}=0
\end{aligned}
$$

1) Optimal packet size $l_{f}^{*}$ and coding rate $v_{f}^{*}$ : Combining Eqns. (2), (3) and (4), the optimal solution satisfies

$$
\begin{aligned}
& \left(1+\frac{2 I_{f}}{\left(1-2 v_{f}\right) \theta_{f}}-p_{f}\right) \\
& \times\left(1+\left(\frac{T_{o}}{T_{c}}-1+\prod_{g \in \mathcal{F}, g \neq f}\left(1+x_{g}\right)\right) \frac{w_{f} T_{c}}{l_{f}}\right)=1
\end{aligned}
$$

In (5), $\left(\frac{T_{o}}{T_{c}}-1+\prod_{g \in \mathcal{F}, g \neq f}\left(1+x_{g}\right)\right) \frac{w_{f} T_{c}}{l_{f}}>0$ and $\frac{2 I_{f}}{\left(1-2 v_{f}\right) \theta_{f}}>$ 0 . Hence at the optimum we must have $p_{f}>0$. By complementary slackness, the constraint on the flow packet size must therefore be tight, i.e. the optimal packet size is

$$
l_{f}^{*}=\bar{l}_{f}
$$

The optimal coding rate $v_{f}^{*}$ can now be found by solving (3).

2) Optimal transmission attempt parameter $x_{f}^{*}$ : It remains to determine the optimal station transmission rate $x_{f}^{*}$. This can be found using standard subgradient descent techniques, e.g. using the algorithm detailed in Alg. 1.

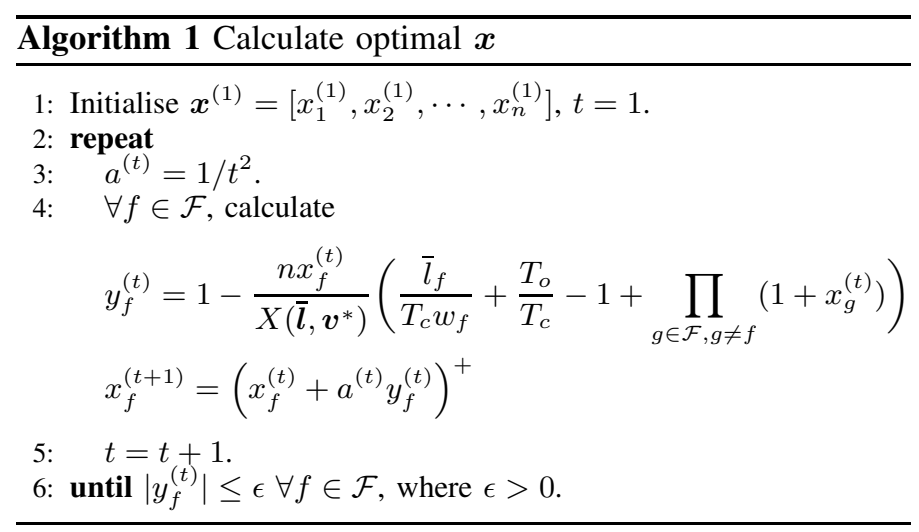

\section{A. Equal air-time}

Due to the presence of collision losses and the coupling of station transmissions via carrier sense, the flow air-time in a WLAN is not simply the successful transmission duration but also includes airtime expended in collisions. Following [8], define the flow total air-time as the fraction of time used for transmissions by flow $f$, including both successful transmissions and collisions, which is given by

$$
\begin{aligned}
t_{f} & =\frac{P_{s, f} T_{s, f}+\left(1-P_{s, f}\right) T_{c}}{\sigma P_{i}+\sum_{f \in \mathcal{F}} T_{s, f} P_{s, f}+T_{c}\left(1-P_{i}-P_{s}\right)} \\
& =\frac{1}{X}\left(\frac{\tau_{f}}{P_{i}}+x_{f}\left(\frac{T_{s, f}}{T_{c}}-1\right)\right)
\end{aligned}
$$

KKT condition (2) can be rewritten as

$$
\frac{1}{X}\left(\frac{\tau_{f}}{P_{i}}+x_{f}\left(\frac{T_{s, f}}{T_{c}}-1\right)\right)=\frac{1}{n}
$$

Hence, the proportional fair allocation assigns equal flow total air-times amongst flows, and the flow total air-times sum to unity. It is worth pointing out that since the flow air-time usage overlaps due to collisions, the flow total air-times summing to unity does not imply that the channel idle probability $P_{i}=0$.

\section{B. Decoupled allocation tasks}

It is the packet size $l_{f}$ that acts to couple the flow coding rate and transmission rate in the optimisation in objective $U_{1}$,

$$
\begin{aligned}
U_{1}(\tilde{\boldsymbol{l}}, \tilde{\boldsymbol{I}}, \tilde{\boldsymbol{x}}) & =\sum_{f \in \mathcal{F}} \underbrace{\tilde{x}_{f}-\log X(\tilde{\boldsymbol{l}}, \tilde{\boldsymbol{x}})+\tilde{l}_{f}}_{\tilde{\boldsymbol{x}} \tilde{\boldsymbol{l}}}-\log T_{c} \\
& +\underbrace{\log \left(1-2 v_{f}(\tilde{\boldsymbol{I}})\right)+\log \left(1-e^{-D_{f} e^{\tilde{\tau}_{f}+\tilde{I}_{f}}}\right)}_{\tilde{\boldsymbol{I}} \tilde{\boldsymbol{l}}}
\end{aligned}
$$

Our analysis establishes that the optimal choice is to select the maximum possible packet size $l_{f}^{*}=\bar{l}_{f}$. Substituting for this known value, $U_{1}$ separates into a term in $\tilde{\boldsymbol{x}}$ and a term in $\tilde{\boldsymbol{I}}$. That is, the optimisation problem decomposes into decoupled flow transmission rate and coding rate allocation tasks. Inspection of KKT condition (3) reveals that the optimal $\boldsymbol{I}$ is dependent on channel condition parameter $\boldsymbol{\beta}$ and delay requirement $\boldsymbol{D}$, while from KKT condition (2) it can be seen that the optimal $\boldsymbol{x}$ is dependent on PHY rate $\boldsymbol{w}$ and the number of stations $n$ in the WLAN. Therefore a layered approach that separates MAC scheduling and packet coding rate selection is optimal.

\section{Decentralised 802.11 implementation}

The optimal $l_{f}^{*}, v_{f}^{*}, x_{f}^{*}$ can be determined by the station carrying flow $f$ without the need for any message passing. Solving (3) to find optimal $v_{f}^{*}$ requires only local information which is available at each station. In Alg. 1 observe that (i) the flow throughput $s_{f}$ can be measured by station $f$, and the quantity $X$ can then be computed based on (1); (ii) the channel idle probability $P_{i}$ can be observed by all stations in a WLAN (via carrier sense, see for example in [9]), and hence we have the quantity $\prod_{g \in \mathcal{F}, g \neq f}\left(1+x_{g}\right)=\frac{1}{P_{i}} \cdot \frac{1}{1+x_{f}}$. Therefore, the update of $x_{f}$ in Alg. 1 can also be carried out locally at each station. 


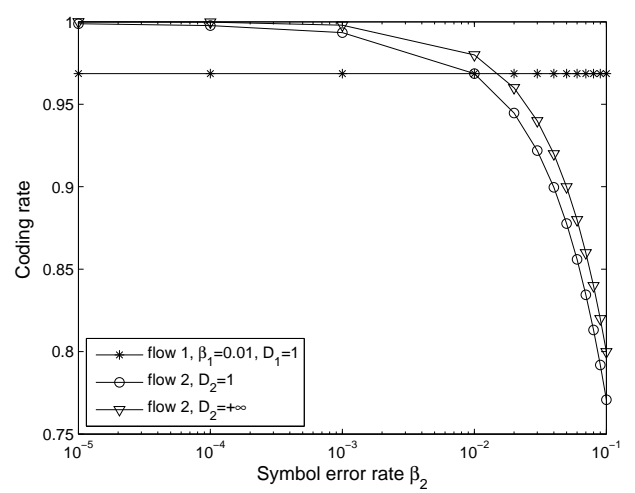

Fig. 1. Single WLAN with 2 flows, packet size $\bar{l}_{1}=\bar{l}_{2}=8000$ bits, PHY rates $w_{1}=w_{2}=54 \mathrm{Mbps}$

\section{EXAMPLES}

\section{A. Impact of $B E R$}

Consider a WLAN with two stations with equal transmitted packet sizes $\bar{l}_{1}=\bar{l}_{2}$ and PHY rates $w_{1}=w_{2}$. The proportional fair allocation assigns equal attempt probabilities to both stations. Fig. 1 plots the optimal coding rates for both flows as the symbol error rate $\beta_{2}$ of flow 2 is varied while keeping $\beta_{1}$ of flow 1 fixed. It can be seen that when the flows have the same delay deadlines, the flow with poorer channel conditions is allocated with a lower coding rate. When the delay deadline for flow 2 is increased to infinity, corresponding to delayinsensitive applications, the coding rate of flow 2 increases. That is, a tighter delay deadline comes at the cost of using a lower coding rate and so lower throughtput - this is to be expected since delay insensitive flows can use a larger code block size and so gain more efficient error protection. It can be also seen that changing the symbol error rate or delay threshold for flow 2 does not affect the coding rate of flow 1. This is because the optimal coding rate for a flow is only dependent on its own symbol error rate and delay deadline.

\section{B. Impact of packet size}

Now consider a WLAN with 6 flows which have identical delay deadlines, symbols error rates and PHY rates, but each flow has a different maximum transmitted packet length. Fig. 2(a) plots the optimal station attempt probability versus the successful transmission durations $T_{s, f}$. Fig. 2(b) plots the corresponding successful flow air-times and flow total airtimes. It can be seen that the proportional fair allocation assigns equal flow total air-times but unequal successful flow air-times. Although flows with longer successful transmission durations are allocated with lower attempt probabilities, they still have longer successful flow air-times. The optimal coding rate for each flow is also different, since the different packet sizes yield different code block sizes (recall the delay deadlines are the same for all flows). That is, neither the optimal coding rate nor the optimal attempt probability is the same for each flow. Similar behaviour occurs when the packet sizes for flows are the same but the PHY rates $w_{f}$ differ.

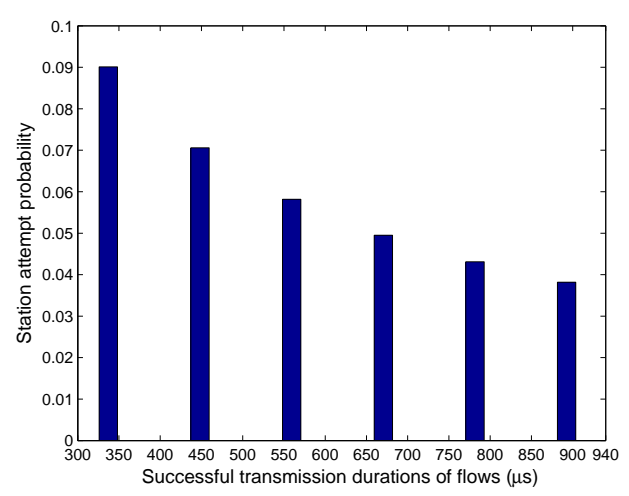

(a) Station attempt probability

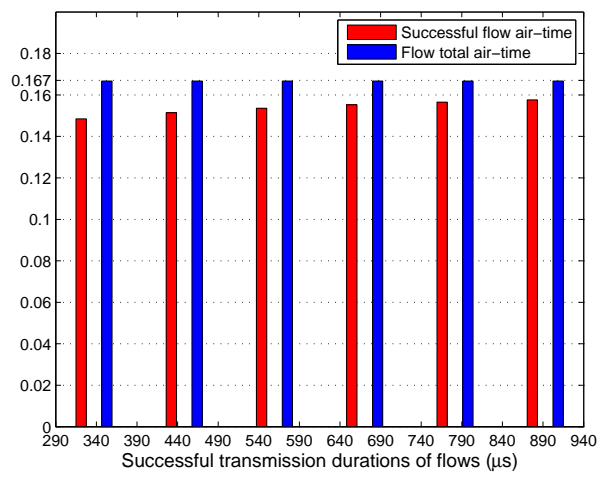

(b) Air-time

Fig. 2. Single WLAN with 6 flows. For each flow, the PHY rate $w=$ $54 \mathrm{Mbps}$, delay deadline $D=1$ and BSC crossover probability $\alpha=10^{-3}$.

\section{CONCLUSION}

We derive the joint allocation of coding rate and airtime that achieves the proportionally fair throughput allocation in an 802.11 WLAN. We show that the joint optimisation decomposes into decoupled allocation tasks and (i) assigns equal total airtime to stations, (ii) selects the station transmission attempt probabilities such that the airtimes sum to unity, (iii) selects the optimal coding to maximise goodput (treating packets decoded after the delay deadline as losses).

\section{REFERENCES}

[1] K. Lin, N. Kushman and D. Katabi,"ZipTx: Harnessing partial packets in 802.11 networks", Proc ACM MobiCom'08, pp. 351-362, 2008.

[2] X. Chen, V.G. Subramanian, D.J. Leith, "Binary Symmetric Channel Based Aggregation with Coding for 802.11n WLANs", Proc. Broadnets, 2010.

[3] D. J. Leith, V. G. Subramanian and K. Duffy, "Log-convexity of rate region in 802.11e WLANs", IEEE Comms Letters, 14(1), pp. 57-59, 2010.

[4] F. J. MacWilliams and N. J. A. Sloane, The theory of error-correcting codes, North-Holland Publishing Co., Amsterdam, 1977.

[5] D. Malone, K. Duffy and D. J. Leith, "Modeling the 802.11 distributed coordination function in nonsaturated heterogeneous conditions", IEEE Trans Networking, 15(1), pp. 159-172, 2007.

[6] K. Premkumar, X. Chen and D. J. Leith, "Proportional fair coding for wireless mesh networks", submitted to IEEE Trans Networking, Jan. 2012.

[7] S. Boyd, L. Vandenberghe, "Convex Optimisation", Cambridge Univ. Press, 2008.

[8] A. Checco, D. J. Leith, "Proportional fairness in 802.11 wireless LANs", IEEE Comms Letters, 15(8), pp. 807-809, 2011.

[9] D. Malone, P. Clifford and D. J. Leith, "MAC layer channel quality measurement in 802.11", IEEE Comms Letters, 11(2), pp. 143-145, 2007. 